\title{
Color flow mapping: A non-invasive tool for characterizing and differentiating between uveal melanomas and choroidal metastases
}

\author{
MEIRA NEUDORFER ${ }^{1}$, MICHAEL WAISBOURD ${ }^{1}$, IRENE ANTEBY ${ }^{3}$, ALON LIRAN ${ }^{1}$, \\ DAFNA GOLDENBERG ${ }^{1}$, ADIEL BARAK $^{1}$ and ADA KESSLER ${ }^{2}$ \\ Departments of ${ }^{1}$ Ophthalmology and ${ }^{2}$ Radiology, Tel-Aviv Sourasky Medical Center, \\ Sackler Faculty of Medicine, Tel-Aviv University, Tel-Aviv; ${ }^{3}$ Department of Ophthalmology, \\ Hadassah-Hebrew University Medical Center, Jerusalem, Israel
}

Received May 20, 2010; Accepted July 5, 2010

DOI: 10.3892/or_00001046

\begin{abstract}
The objective of this study was to characterize and differentiate vascular patterns of choroidal melanomas and choroidal metastases by color flow mapping (CFM). We conducted a retrospective chart study on CFM findings in 18 patients with choroidal melanomas and 10 patients with choroidal metastases. We evaluated the possibilities of applying CFM to identify hypo- versus hyper-vascularity within the tumor, a central 'dominant vessel', and Doppler signals in the tumor's center and/or periphery. CFM demonstrated hypervascular patterns in 33\% melanomas and $100 \%$ metastases $(\mathrm{p}<0.0001)$. CFM identified a central dominant vessel in 94\% melanomas and $0 / 10$ metastases $(p<0.0001)$. Vascularity occupied the center of 11/18 melanomas and $0 / 10$ metastases $(p<0.0001)$, the periphery of $2 / 18$ melanomas and $9 / 10$ metastases $(\mathrm{p}<0.0001)$, and equally occupied the tumor's center and periphery of 5/18 melanomas and 1/10 metastases $(p<0.0001)$. Based on our findings, we conclude that CFM was capable of non-invasively demonstrating different and distinct vascular patterns in malignant choroidal melanomas and choroidal metastases.
\end{abstract}

\section{Introduction}

The diagnosis of intraocular tumors is often based on clinical characteristics. The clinical differentiation between primary choroidal melanoma and choroidal metastases may pose a diagnostic challenge in patients with atypical findings. In some centers, fine-needle biopsy (FNB) of the tumor is used to distinguish melanoma from metastases when the com-

Correspondence to: Dr Meira Neudorfer, Department of Ophthalmology, Tel-Aviv Medical Center, 6 Weizman Street, Tel-Aviv 64239, Israel

E-mail: meiraneu@netvision.net.il

Key words: color flow mapping, choroidal melanoma, choroidal metastasis prehensive medical workup is non-definitive. When available and technically possible, FNB is certainly the preferred method for reliable diagnosis of intraocular tumors (1). This procedure, however, has a few important limitations, among them the necessity of an experienced cytologist for interpretation and the possibilities of intraocular damage (including vitreous hemorrhage and retinal detachment), inadequacy of the obtained material for the purposes of diagnosis, delay in diagnosis and treatment, and higher cost. Therefore, there is a need for a reliable and risk-free diagnostic auxiliary tool in the clinical assessment of different types of intraocular tumors.

Choroidal melanoma is the most common primary intraocular malignancy, with an incidence of 5-7 cases per one million people and a prevalence of 1200-1500 new cases each year in the United States and Western Europe (2-4). Choroidal metastases are the most frequent intraocular malignancy in the elderly population. The primary tumors that send ocular metastases are breast carcinoma (contributing $65 \%$ of all ocular metastases) followed by lung, kidney, colon and genitourinary malignancies. Clinically, small melanomas ( $<3 \mathrm{~mm}$ in height) and metastases to the choroid may appear as solid amelanotic raised lesions, making the differential diagnosis a difficult task. These small amelanotic lesions may also resemble benign intraocular lesions such as choroidal nevi.

The most widely used diagnostic imaging tool for intraocular tumors is ultrasonography. Standardized ultrasonography by means of A-scan and B-scan modes helps in localizing and estimating the size of an intraocular mass. In addition, a fairly reliable ultrasonic differentiation can be made by characterizing its tissue texture (2-6). Choroidal melanoma is characterized by a dome-shaped mass on a $\mathrm{B}$-scan and by low-to-medium homogeneous internal reflectivity on an A-scan or a good quality grayscale B-scan. It is well recognized that necrosis of a melanoma is expressed by lower homogeneous internal reflectivity (1-4). Choroidal metastases are characterized by a flatter or only slightly dome-shaped mass on a B-scan, they are mostly multilobular and they occasionally have an irregular surface. An A-scan typically shows medium-to-high mostly nonhomogeneous internal reflectivity (2-5). 
Color flow mapping (CFM) demonstrates blood flow on the background of an anatomic ultrasound image (7). It is a relatively inexpensive diagnostic tool and one associated with minimal discomfort. It is a rapid and efficient method which may be used in eyes with both clear and opaque media. Parameters of CFM include: presence of flow, subjective characterization of hypo- versus hyper-vascularity (arbitrary fixed as $<3$ blood vessels within the tumor or $\geq 3$ blood vessels, respectively), and determination of a vascular pattern as 'peripheral' when the vessels are mostly on the periphery of the lesion or 'central' when vessels are mostly in its center. The blood flow on the surface of the lesion typically demonstrated retinal blood flow.

Pulse Doppler examination enables the evaluation of the velocity of flow [peak systolic velocity (PSV) and enddiastolic velocity (EDV)], and thus calculation of the resistance of the flow downstream. The resistance of flow is based on automatic calculation of a 'resistance index' (RI = PSV-EDV/PSV). This index, however, is highly prone to errors due to the extremely low EDV and, therefore, may not be reliable in measuring ophthalmic tumor blood flow.

We now evaluated both vascular patterns and quantitative parameters using CFM in order to help differentiate between primary malignant melanoma and metastases of the choroid. To our knowledge, this is the first application of qualitative vascular patterns on CFM for the purpose of differentiating between the two pathologies. The parameters of vascular patterns of the tumors that we investigated were: i) hypoversus hypervascularity; ii) identification of a 'dominant vessel'; and iii) characterization of blood distribution within the tumor, e.g., central versus peripheral blood flow.

\section{Materials and methods}

We retrospectively evaluated eyes of consecutive patients with intraocular tumors attending the ophthalmology outpatient clinic of the Tel Aviv Sourasky Medical Center. Patient data and ultrasonic parameters were retrieved from the hospital charts. During their initial evaluation, all patients underwent a comprehensive ophthalmic examination, including fundus photography of the intraocular tumor. A detailed history for the presence of metastases was taken for all participants. The patients were also evaluated by the oncologist with suitably selected imaging and blood tests. All the patients underwent CFM after providing informed consent. The physician performing the CFM was not masked to the diagnosis of the intraocular tumor.

Color flow mapping. CFM scanning was performed by the same physician (A.K.) with Acuson XP10 (Mountain View, CA, USA) and ATL Philips HDI 5000 (Seattle, WA, USA) scanners using 7.5 MHz or 5-12 MHz linear transducers, respectively. B-mode and Doppler examinations were performed in axial and oblique planes. The examinations were performed with the patient in the supine position, both eyes closed, while looking straight ahead. Additional lateral or medial views were performed to gain a better visualization of the ciliary blood vessels. Gel was applied on the closed eyelid, and the hands of the examiner were placed on the borders of the orbits in order to avoid elevation of intraocular pressure. The gray scale examination demonstrated the anatomical details of the tumor and its sonographic characteristics, while the CFM examination revealed the vascular pattern within the tumor.

The CFM settings were for high sensitivity with low wall filter to allow detection of vessels with low blood flow. The pulse Doppler gate was reduced to a sample of $1 \mathrm{~mm}$. Characterization of the tumors was done based on quantitative and qualitative parameters. Quantitative parameters included vascular resistance, which was evaluated at different sites within the vessels by three consecutive Doppler spectral wave-forms recordings. Additional parameters that were used for this evaluation included PSV, EDV and RI. The evaluation of the qualitative parameters included: i) hypo-vascularity or hyper-vascularity within the tumor that was arbitrary fixed as $<3$ blood vessels within the tumor or $\geq 3$ blood vessels, respectively; ii) demonstrating a larger vessel (therefore called 'dominant') entering the central part of the tumor (this term should not be confused with the histological description of feeder vessel); and iii) the 'vascular patterns' of the ocular tumors, as defined by CFM. These were classified into three main patterns depending on the location of the vascularization within the tumor: 'central' when the flow signals appeared mainly in the center of the tumor mass, 'peripheral' when the flow signals appeared around the periphery of the tumor mass and 'mixed' when the flow signals appeared both within the tumor mass and along its borders.

Statistical analysis. The data were analyzed using the $\chi^{2}$ test to evaluate the relations between the tumor diagnosis and the results of the CFM test. Analysis by t-test and Mann-Whitney test was performed to compare between the quantitative means measured. All calculations were performed using SPSS statistical software.

The study was performed with the approval of the institutional ethics committee.

\section{Results}

We evaluated 28 eyes of 27 consecutive patients with intraocular tumors. Eighteen eyes of 18 patients had intraocular tumors diagnosed as choroidal malignant melanoma, and 10 eyes of 9 patients had choroidal metastases. The mean age of the patients was 56.6 years (range 17-80) in the melanoma group and 58.1 years (range 47-86) in the metastases group. Tumor size varied: it ranged between $2.5-13 \mathrm{~mm}$ (mean $7.5 \mathrm{~mm}$ ) in the melanoma group and between $2.5-8 \mathrm{~mm}$ (mean $4.4 \mathrm{~mm}$ ) in the metastases group. Three eyes had choroidal melanomas with height $<3 \mathrm{~mm}$. CFM could be demonstrated even in the smallest lesions of $2.5 \mathrm{~mm}$ in both groups. Tumor size did not affect our results. Two of the melanomas were mushroom-shaped. There was no difference between the vascular patterns of these mushroom-shaped melanomas and the dome-shaped group.

The diagnosis was made by clinical and ultrasonic features alone in 12 of the 18 eyes (67\%) with choroidal melanoma. According to the first report of the Collaborative Ocular Melanoma Study (COMS), choroidal melanoma was mis- 


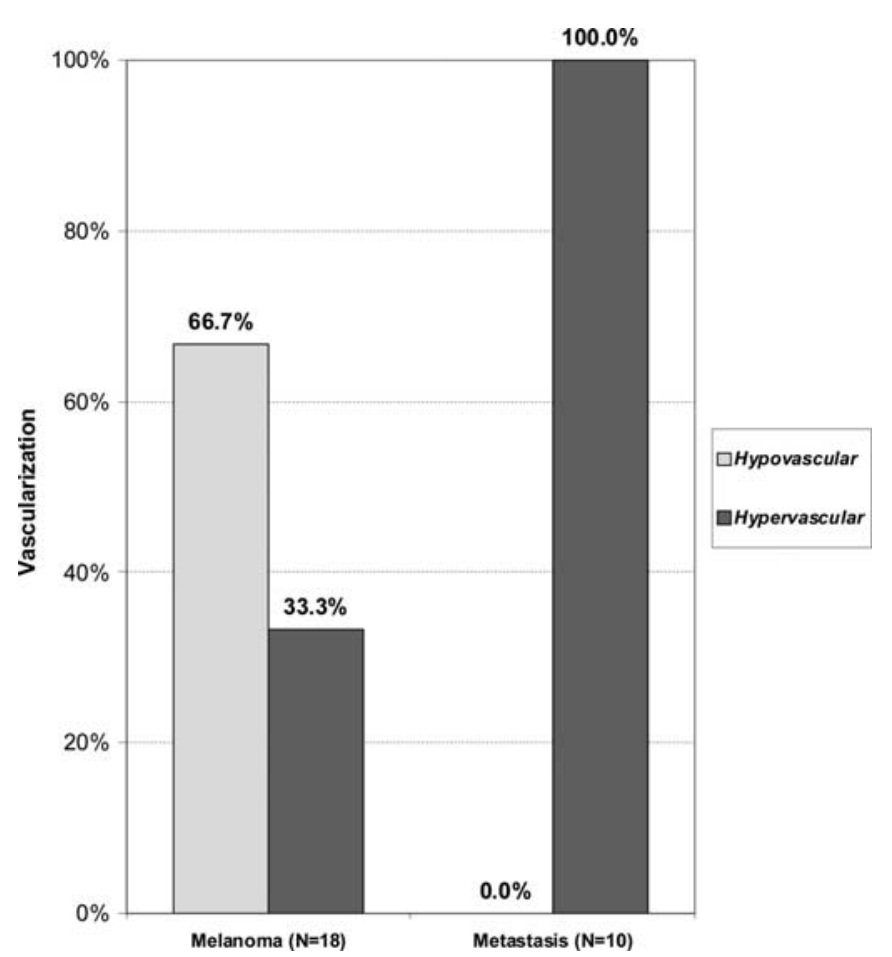

Figure 1. Two-thirds of the choroidal malignant melanomas showed 'hypovascularity' of the tumors, while choroidal metastases had solely 'hypervascularity' in all tumors.

diagnosed in only $0.48 \%$ of cases based on these parameters alone (8-10). In addition, the diagnosis of choroidal melanoma was reinforced by a typical response to brachytherapy. Furthermore, a prolonged follow-up period (mean = 26.8 months) of all melanoma patients yielded no evidence for metastases or any other non-ocular malignancy. Histopathological diagnosis of melanoma was not performed in these 12 eyes, either due to inaccessibility or to the patient's or physician's choice. Diagnosis of malignant melanoma of the uvea was confirmed by pathological tissue examination after enucleation of the tumor due to its size on presentation in $7 / 18$ eyes $(38 \%)$.

Eleven of the patients diagnosed with choroidal melanoma were treated by brachytherapy, and 7 patients underwent enucleation. All ten choroidal metastases were diagnosed according to the typical clinical features together with ocular ultrasound characteristics in the context of systemic evidence of a primary non-ocular tumor. The final diagnosis of the tumor's origin was diagnosed as breast $(n=6)$, lung $(n=2)$, rectum $(n=1)$ and stomach $(n=1)$. Melanomas with subretinal fluid had the same CDI characteristics as those without. The mean follow-up of patients who had metastases was 113 months (range 2-31). The CFM results of 28 intraocular tumors of the 13 male and 14 female study participants are reported.

CFM quantitative parameters. The mean EDV was $4.5 \mathrm{~cm} / \mathrm{sec}$ (range 1-15) in the melanoma group and $7.1 \mathrm{~cm} / \mathrm{sec}$ (range $2-20)$ in the metastases group $(\mathrm{p}=0.11)$. The mean PSV was $12.9 \mathrm{~cm} / \mathrm{sec}$ (range 3-36) in the melanoma group and 19.1 $\mathrm{cm} / \mathrm{sec}$ (range 5-57) in the metastases group $(\mathrm{p}=0.25)$. The

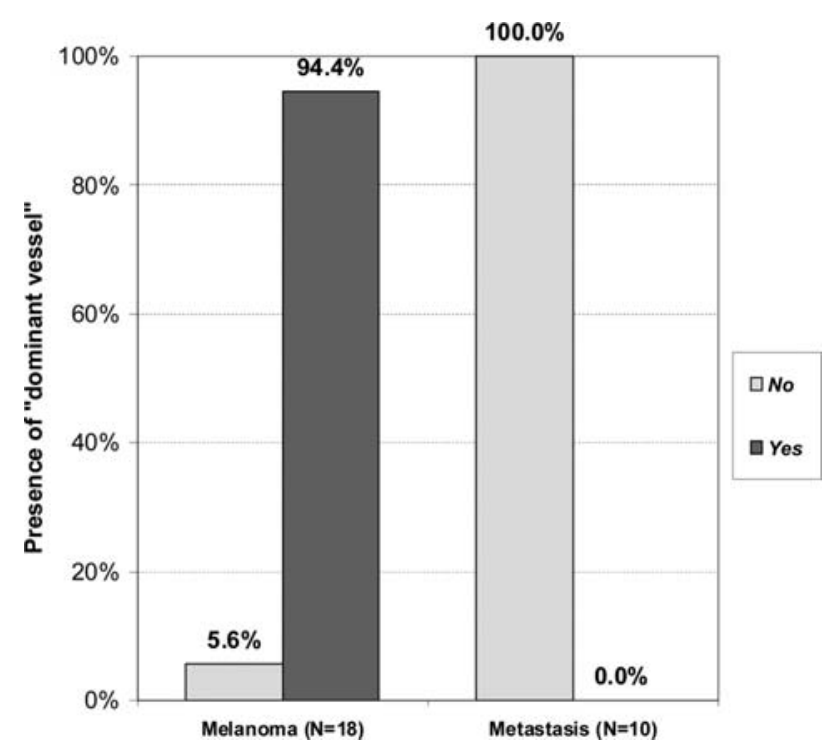

Figure 2. Almost all choroidal malignant melanomas had a central 'dominant vessel', which was entirely absent from the choroidal metastases.

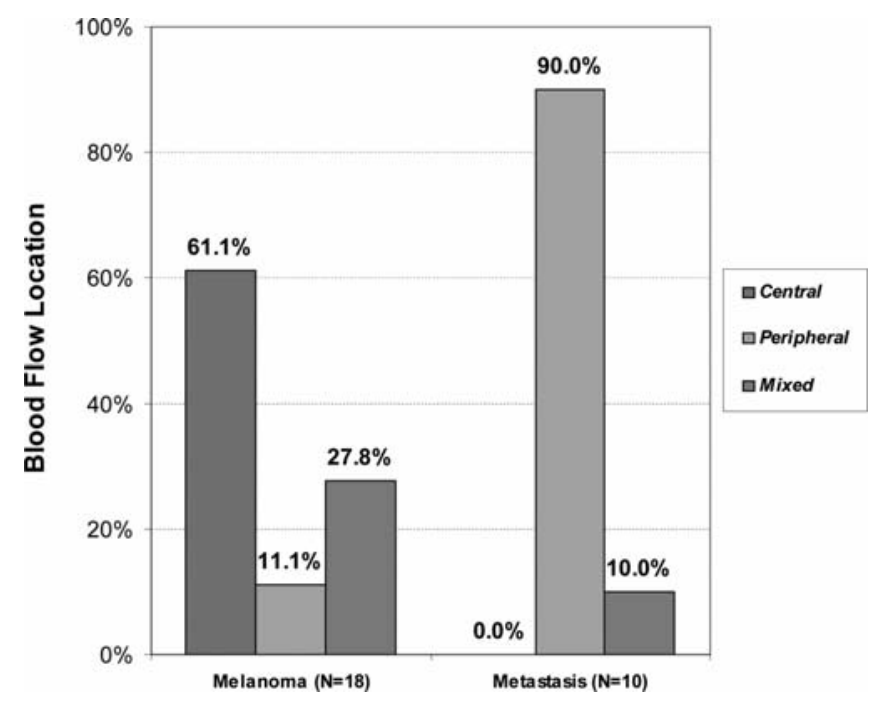

Figure 3. Blood vessel distribution was mainly of central or mixed pattern in choroidal melanomas, whereas $90 \%$ of choroidal metastases showed a peripheral pattern of blood flow and no central distribution whatsoever.

mean RI was 0.7 (range 0.5-0.8) in the melanoma group and 0.6 (range $0.5-0.7)$ in the metastases group $(\mathrm{p}=0.33)$.

CFM qualitative parameters. Two-thirds (12/18, 67.7\%) of the tumors in the melanoma group showed hypovascularity and one-third $(6 / 18,33.3 \%)$ showed hypervascularity within the tumor. In the metastases group, all 10 tumors showed hypervascularity. This difference reached a level of significance $(\mathrm{p}<0.001)$ (Fig. 1). All but one of the tumors $(17 / 18$, $94.4 \%)$ in the melanoma group demonstrated central flow with a dominant vessel. None of the 10 tumors in the metastases group demonstrated a dominant vessel. This difference was also significant $(\mathrm{p}<0.001)$ (Fig. 2). Eleven $(11 / 18,61.1 \%)$ of the tumors in the melanoma group showed 
A

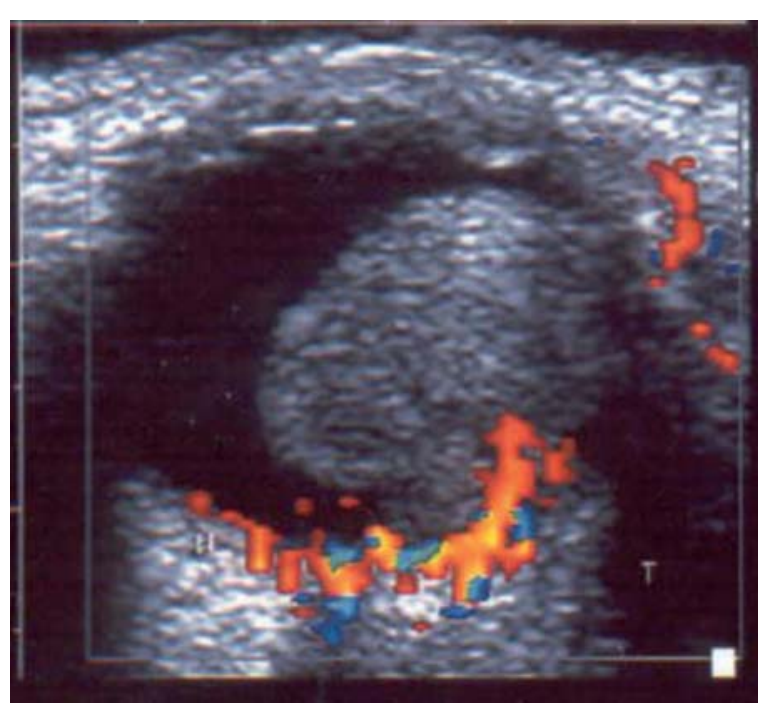

C

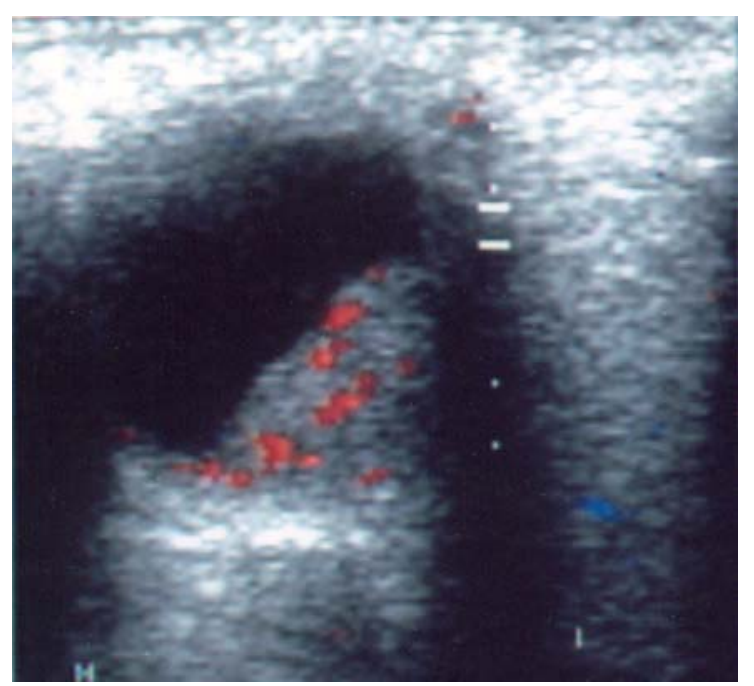

B

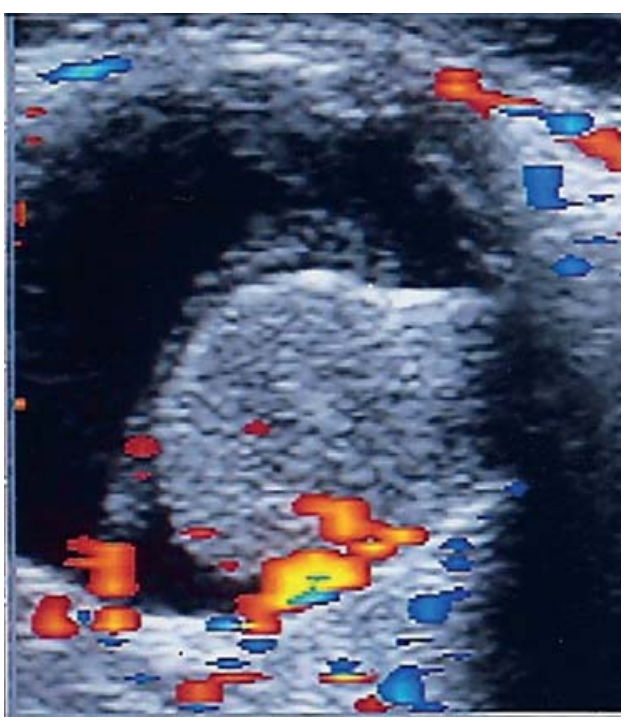

D

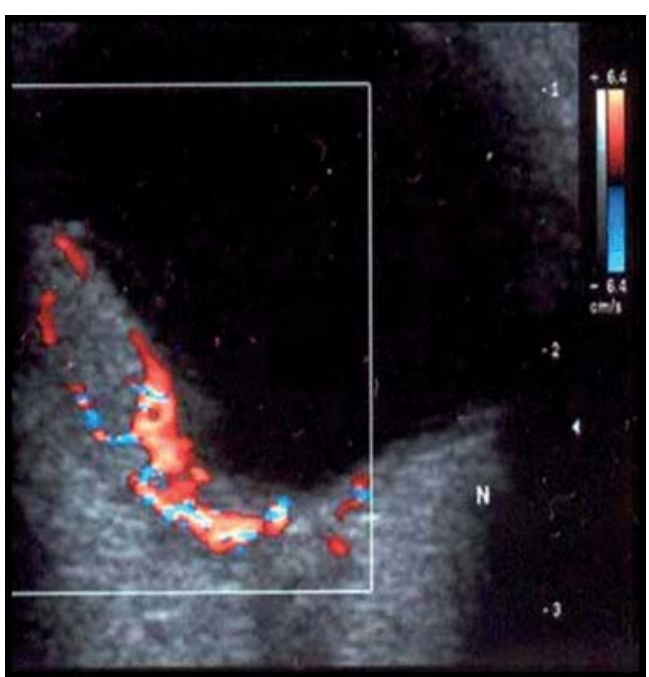

Figure 4. Qualitative parameters on color Doppler imaging differentiating between melanomas and metastases to the choroid. (A and B) Choroidal melanomas showing the presence of a central 'dominant vessel' with no peripheral blood vessels, therefore characterized as 'hypovascular'. (C and D) Choroidal metastases showing multiple blood vessels within the tumor, therefore characterized as 'hypervascular'. There is no 'dominant vessel' and the blood flow distribution is of a mixed type.

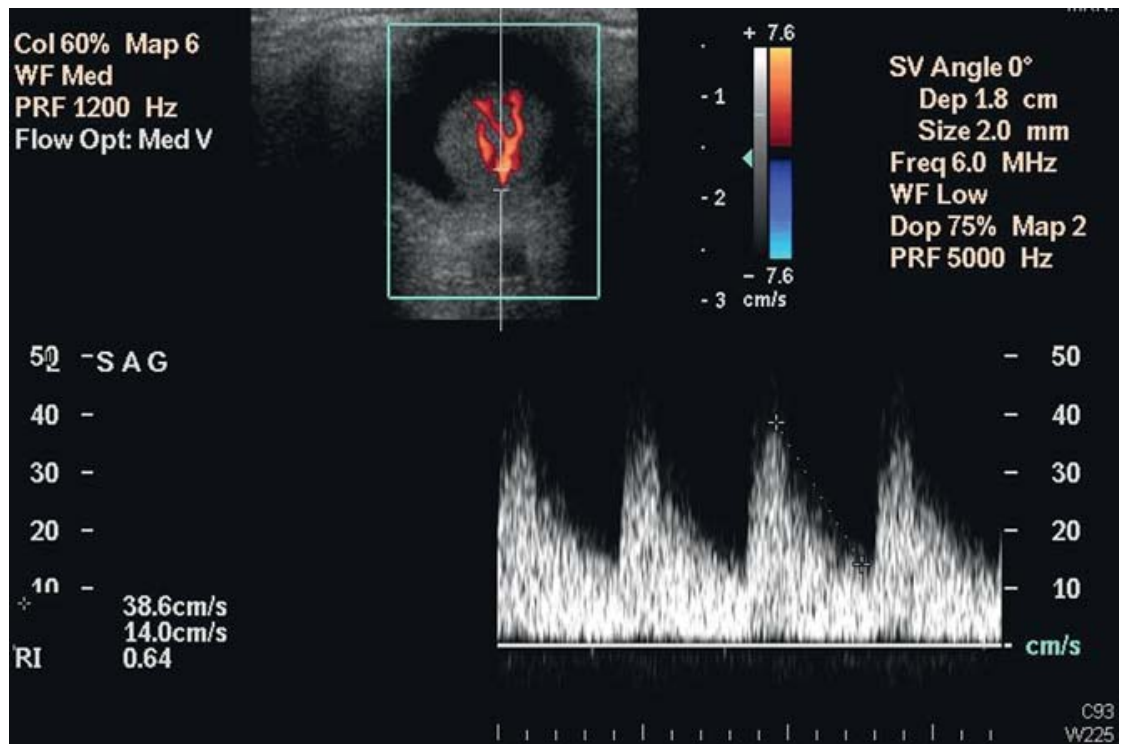

Figure 5. Choroidal melanoma, qualitative and quantitative parameters. Color flow map with combined spectrogram. 
a 'central pattern', 2 (11.1\%) showed 'peripheral pattern', and $5(27.8 \%)$ had a 'mixed pattern' of blood flow. None of the 10 tumors in the choroidal metastases group had a 'central pattern', nine (90\%) had a pure 'peripheral pattern' and one (10\%) showed a 'mixed pattern' of blood flow. This difference was significant ( $\mathrm{p}<0.001)$ (Fig. 3). Fig. 4 summarizes the differences in vascular patterns between choroidal melanomas and choroidal metastases. Fig. 5 demonstrates qualitative and quantitative parameters.

\section{Discussion}

The clinical differentiation between choroidal melanomas and choroidal metastases is often straight-forward. Atypical cases may, however, present a diagnostic challenge and require adjunctive tools to help differentiate between the tumors. The 'gold standard' method for final diagnosis of a tumor mass is FNB, which is regularly carried out in some centers by ophthalmic oncologists worldwide, even though it is an invasive procedure that may be devastating for the integrity of the eye and for vision when it is found at certain intraocular tumor locations. Thus, the search for non-invasive and injury-free methods to minimize risks for patients who have an atypical appearance of intraocular tumors. Conventional methods of diagnosis are based on only the clinical and ultrasonic characteristics of the mass. Our study results clearly show the advantage of a novel approach to distinguishing between choroidal melanomas and choroidal metastases by means of CFM. The additional information obtained by this method may contribute to the differential diagnosis when tissue sampling is not a viable option.

The application of CFM is well established in a variety of conditions, among them evaluating the patency of vessels and organs and for demonstrating vascular malformation, inflammation and tumors. The CFM is estimated subjectively as either no flow, hypo-vascularity or hyper-vascularity. There is no calculation of the number of vessels per area. Many diagnoses, such as testicular torsion, orchitis, epididymitis (11), thyroiditis (12), lymphadenitis versus metastatic disease in lymph nodes (13) and synovitis (14), are made solely on subjective color Doppler estimations. Although tumors can be characterized as being hypo- or hyper-vascular, CFM is not specific and additional imaging or histologic evaluation is usually needed $(15,16)$. Tumors have also been evaluated by CFM characterizations, such as the absence of vascularization, presence of peripheral vascularization and presence of central vascularization, by means of methods similar to the one used in the current study (17).

CFM has previously been used for distinguishing between choroidal melanomas and metastases by looking solely at quantitative parameters. Wolff-Korman et al (18) used CFM for the differential diagnosis of choroidal tumors. Out of 103 untreated choroidal tumors, the mean PSV of choroidal melanomas $(n=62$, mean $P S V=0.95 \mathrm{kHz})$ was significantly lower than that of choroidal metastases $(n=12$, $\mathrm{PSV}=1.87 \mathrm{kHz}$ ). Those authors concluded that quantitative measurement of tumor blood flow by CFM may serve as a diagnostic tool in the evaluation of intraocular tumors. Lieb et al (19) demonstrated abnormal Doppler shifts within all
39 studied neoplastic lesions, but these shifts could not be detected in three tumor-simulating lesions. Those authors concluded that CFM may be of value as an additional diagnostic tool in the diagnosis and management of intraocular tumors. CFM was also used as a diagnostic modality for monitoring the effectiveness of radiotherapy in choroidal melanoma by showing a decrease in blood flow signals to the tumor after radiotherapy $(19,20)$.

We now introduce a qualitative evaluation of the tumor's vascular characteristics. This additional information enhances the certainty of diagnosis and does so by noninvasive means, as part of the assessment of tumors clinically diagnosed as choroidal melanomas or metastases.

Our current study introduces new qualitative imaging features that reflect the vascular behavior of the two tumor entities. We identified tumors with hypo-vascularity, i.e., demonstrating fewer than 3 vessels per tumor, as opposed to hyper-vascularity, i.e., demonstrating more than 3 vessels. Furthermore, we established the presence or absence of a 'dominant' centrally located vessel within the tumor mass. Finally, we located the main blood flow of the tumor as being central, peripheral or of a mixed type. These qualitative features form a distinct pattern for contributing to the differentiation between melanomas and metastases. We demonstrated that choroidal melanomas typically have vascular characteristics, including that of hypovascularity, with a dominant centrally located vessel and with a 'central pattern' blood flow. In contrast, choroidal metastases tend to have hyper-vascularity, lack a 'dominant vessel' and typically have a 'peripheral pattern' blood flow. These differences may be explained by several theories: given that neovascularization is the cornerstone of tumor proliferation, their being related to the nature of these tumors may be the more plausible among them. Tumor cells secrete neovascular factors in order to allow the supply of nutrients for the developing tumor. The extent of tumor vasculature is directly related to its risk to spread. For example, endothelial cells may proliferate at a rate of up to 100 times more than their baseline proliferation rate during neovascularization (21). The extent of blood vasculature in a tumor depends upon a process of the sprouting of new blood vessels as well as on the deviation of existing blood vessels to the tumor area (19). Angiogenic factors were recently found to be expressed in uveal melanomas, including isoforms of vascular endothelial growth factor (VEGF)-A to D (22), and an anti-angiogenic therapy is currently being investigated as a possible future treatment option (23).

Interestingly, when looking at standard CFM quantitative parameters, such as PSV, EDV and IR, we did not find any significant differences between the two tumor groups. As such, our findings are not consistent with those of WolffKormann et al (20). Specifically, while we did not find these parameters useful for distinguishing between choroidal melanomas and metastases, Wolff-Korman et al (20) concluded that quantitative measurement of tumor blood flow by CFM may serve as a diagnostic tool in the evaluation of intraocular tumors.

This study has a few important limitations: it was conducted retrospectively, and the physician performing the CFM was not masked to the diagnosis (melanoma or meta- 
stasis). It is partly qualitative and subjective and, therefore, may be dependent on researcher interpretation. There was also a lack any patients in the diagnosis uncertain group. The rather small number of cases and the range of primaries are the major limitations of this study. However, the unique presentation of different vascular patterns of choroidal melanomas versus metastases presented herein may lay the foundations for future prospective and masked studies. Such studies are needed to verify the results that had been obtained in this study.

Moreover, hypo-vascularity had been defined as fewer than 3 vessels within the tumor, whereas hyper-vascularity was defined as more than 3 vessels: this classification does not take into account the size or volume of the tumor. Hence, a large tumor with 4 vessels may actually be less vascular than a small tumor with 3 vessels. Pulsation of tumor vessels was not directly observed: when evaluating large vessels, pulsation can be seen even in the gray scale image by looking at the arterial wall, however, the tumoral blood vessels in the eye are too small to show this feature and their flow is evaluated by duplex characteristics of high and low resistance pulsatility of the flow. High pulsatility means high resistance in the tumor, low pulsatility means low resistance.

To conclude, we suggest that CFM is a useful and feasible supplementary non-invasive diagnostic tool in the differentiation between atypical cases of primary melanomas and secondary metastases to the choroid. The new qualitative parameters seen on CFM described herein can help in the challenging task of differentiating between these two tumors when presentation is atypical.

\section{Acknowledgements}

Esther Eshkol is thanked for editorial assistance.

\section{References}

1. Cohen VM, Dinakaran S, Parsons MA and Rennie IG: Transvitreal fine needle aspiration biopsy: the influence of intraocular lesion size on diagnostic biopsy result. Eye 15: 143-147, 2001.

2. Char DH: Clinical ocular oncology. Churchill Livingstone Inc., New York, 1989.

3. Anteby I and Pe'er J: [Ultrasonography-a major imaging tool in the diagnosis of intraocular tumors]. Harefuah 124: 96-100, 1993.

4. Peer J: [Uveal malignant melanoma]. Harefuah 124: 40-44, 1993.

5. Frazier Byrne $S$ and Green R: Ultrasound of the Eye and Orbit. Mosby-Year Book Inc., St. Louis, 1992.
6. Sobottka B, Schlote T, Krumpaszky HG and Kreissig I: Choroidal metastases and choroidal melanomas: comparison of ultrasonographic findings. Br J Ophthalmol 82: 159-161, 1998.

7. Belden CJ, Abbitt PL and Beadles KA: Color Doppler US of the orbit. Radiographics 15: 589-608, 1995.

8. Echography (Ultrasound) Procedures for the Collaborative Ocular Melanoma Study (COMS), Report no. 12, Part II. J Ophthalmic Nurs Technol 18: 219-232, 1999.

9. Echography (Ultrasound) Procedures for the Collaborative Ocular Melanoma Study (COMS), Report no. 12, Part I. J Ophthalmic Nurs Technol 18: 143-149, 1999.

10. The Collaborative Ocular Melanoma Study: Accuracy of diagnosis of choroidal melanomas. COMS report no. 1. Arch Ophthalmol 108: 1268-1273, 1990.

11. Schalamon J, Ainoedhofer H, Schleef J, Singer G, Haxhija EQ and Höllwarth ME: Management of acute scrotum in children the impact of Doppler ultrasound. J Pediatr Surg 41: 1377-1380, 2006.

12. Saleh A, Santen R, Malms J, Feldkamp J, Fürst G, Scherbaum WA and Mödder U: [B-mode ultrasound and modern Doppler ultrasound methods in diseases of the thyroid gland and parathyroid glands]. Radiologe 38: 344-354, 1998.

13. Issing PR, Kettling T, Kempf HG, Heermann R and Lenarz T: [Ultrasound evaluation of characteristics of cervical lymph nodes with special reference to color Doppler ultrasound. A contribution to differentiating reactive from metastatic lymph node involvement in the neck]. Laryngorhinootologie 78: 566$572,1999$.

14. Joshua F, Edmonds J and Lassere M: Power Doppler ultrasound in musculoskeletal disease: a systematic review. Semin Arthritis Rheum 36: 99-108, 2006.

15. Clark KJ, Cronan JJ and Scola FH: Color Doppler sonography: anatomic and physiologic assessment of the thyroid. J Clin Ultrasound 23: 215-223, 1995.

16. Watermann D, Madjar H, Sauerbrei W, Hirt V, Prömpeler H and Stickeler E: Assessment of breast cancer vascularisation by Doppler ultrasound as a prognostic factor of survival. Oncol Rep 11: 905-910, 2004.

17. Varverakis E, Neonakis E, Tzardi M and Chrysos E: Role of color Doppler ultrasonography in the preoperative management of cold thyroid nodules. Hormones (Athens) 6: 44-51, 2007.

18. Wolff-Kormann PG, Kormann BA, Hasenfratz GC and Spengel FA: Duplex and color Doppler ultrasound in the differential diagnosis of choroidal tumors. Acta Ophthalmol (Suppl.) 204: 66-70, 1992.

19. Lieb WE, Cohen SM, Merton DA, Shields JA, Mitchell DG and Goldberg BB: Color Doppler imaging of the eye and orbit. Technique and normal vascular anatomy. Arch Ophthalmol 109: 527-531, 1991.

20. Wolff-Kormann PG, Kormann BA, Riedel KG, Hasenfratz GC, Stefani FH, Spengel FA and Lund OE: Quantitative color Doppler imaging in untreated and irradiated choroidal melanoma. Invest Ophthalmol Vis Sci 33: 1928-1933, 1992.

21. Fauci AS, Braunwald E, Isselbacher KJ, Wilson JD, Martin JB and Kasper DL: Harrison's Principles of Internal Medicine. McGraw-Hill Inc., New York, 1998.

22. Notting IC, Missotten GS, Sijmons B, Boonman ZF, Keunen JE and van der Pluijm G: Angiogenic profile of uveal melanoma. Curr Eye Res 31: 775-785, 2006.

23. Rosenblatt MI and Azar DT: Anti-angiogenic therapy: prospects for treatment of ocular tumors. Semin Ophthalmol 21: 151-160, 2006. 